\title{
Retrospektive GKV-Versorgungsforschungsstudie über GnRH-Antagonisten/-Agonisten zur initialen Therapie des fortgeschrittenen Prostatakarzinoms - Verordnungsmuster und Krankenhauskosten in Deutschland
}

\author{
Retrospective SHI (Statutory Health Insurances) real-world study \\ on initial GnRH antagonist and agonist therapy for advanced pro- \\ state cancer: prescription patterns and hospital costs in Germany
}

다(1)잉

Autoren

Marie Christine Hupe', Peter Hammerer², Miriam Ketz ${ }^{3}$, Nils Kossack ${ }^{4}$, Christiane Colling ${ }^{5}$, Axel S. Merseburger ${ }^{1}$

Institute

1 Klinik für Urologie, Universitätsklinikum SchleswigHolstein, Campus Lübeck, Lübeck, Deutschland

2 Klinik für Urologie, Akademisches Lehrkrankenhaus Braunschweig, Braunschweig, Deutschland

3 D-to-D - Data to Decision - AG, Hamburg, Deutschland

4 WIG $^{2}$ - Wissenschaftliches Institut für

Gesundheitsökonomie und

Gesundheitssystemforschung - GmbH, Leipzig,

Deutschland

5 Ipsen Pharma GmbH, München, Deutschland

Schlüsselwörter

fortgeschrittenes Prostatakarzinom,

Versorgungsforschungsstudie, GnRH-Agonist, GnRH-

Antagonist, Androgendeprivationstherapie

Keywords

advanced prostate cancer, retrospective health services

research, GnRH agonist, GnRH antagonist, German claims

database, androgen deprivation therapy

Bibliografie

DOI https://doi.org/10.1055/a-1018-1651 |

Online-Publikation: 19.11.2019 | Akt Urol 2020; 51: 275-284

(c) Georg Thieme Verlag KG Stuttgart · New York

ISSN 0001-7868

Korrespondenzadresse

Dr. Marie C. Hupe, Klinik für Urologie, Universitätsklinikum

Schleswig-Holstein, Campus Lübeck,

Ratzeburger Allee 160, 23538 Lübeck

Tel.: 004945150043601

Fax: 004945150043604

mariechristine.hupe@uksh.de

\section{ZUSAMMENFASSUNG}

Hintergrund Die Androgendeprivationstherapie (ADT) ist fester Therapiebestandteil des fortgeschrittenen oder metastasierten Prostatakarzinoms (PCa). Ziel der vorliegenden Versorgungsforschungsstudie war der Vergleich von Verordnungsmustern, Hospitalisierungsraten und Krankenhauskosten beim initialen Einsatz verschiedener $\mathrm{GnRH}$ Agonisten und -Antagonisten ( $\mathrm{GnRHa}$ ).

Material und Methoden Anonymisierte GKV-Daten von $>70$ Krankenkassen aus 2010 bis $2015(n=4205227)$ wurden analysiert ( 1 Jahr Vorbeobachtung, 1 Indexquartal mit initialer GnRHa-Verordnung, 22 Jahre Follow-Up (FU)).

Ergebnisse Die Studienpopulation umfasste 2382 PCa-Patienten im Alter von durchschnittlich 75 Jahren. Leuprorelin (Leu) wurde mit 56,6\% am häufigsten verordnet. Bei erstmaliger GnRHa-Anwendung hatten 70\% aller Patienten keine Lymph- oder Fernmetastasen. Nach der initialen Verordnung wurden bei $11,2 \%$ die GnRHa abgesetzt, bei 17,6\% nach durchschnittlich 457 Tagen (Median: 399 Tage) umgestellt, in der Hybrid (Hyb)-Gruppe durchschnittlich 100 Tage schneller als bei den Agonisten $(p=0,016)$. Die Prävalenzrangfolge der häufigsten Komorbiditäten war über die Zeit gleichbleibend: Hypertonie, Hyperlipidämie, kardiovaskuläre Erkrankungen (CVD) und Diabetes. Die Hypertonieprävalenz zeigte einen signifikant höheren Anstieg unter Agonisten (16,4\%; vs. Antagonist 6,9\% p=0,022; vs. Hybride $11,6 \% p=0,006)$. Bei der CVD gab es hinsichtlich der relativen Wachstumsraten zwischen den 3 zusammengefassten Therapieklassen keine signifikanten Unterschiede. Insgesamt waren 23,9\% aller Patienten nach 3 Jahren FU verstorben. Die Mortalitätsrate war am niedrigsten für Triptorelin (Trp, 22,1\%) und am höchsten für Goserelin (Gos, $29,4 \%$, n.s.). Im Indexquartal hatten $26,4 \%$ der Patienten mind. einen Krankenhausaufenthalt [min; max: Trp 22,4\%; Gos 30,3\%] mit einer mittleren Krankenhausverweildauer/ Patient von 3 Tagen [Trp 2,4; Gos 4,5]. Die jährliche Hospi- 
talisierungsrate lag bei 36,2-40,7\%, die mittlere Krankenhausverweildauer im gesamten FU zwischen 17,6 (Trp) und 20,8 (Hyb) Tagen. Die Krankenhauskosten im Indexquartal betrugen ca. 1200 EUR [Trp 988; Gos 1803] und pro FUJahr ca. 3000 EUR. In der Trp-Kohorte lagen die Gesamtkosten (Indexquartal+3 Jahre) mehr als 1000 EUR unter dem Durchschnitt von 9476 EUR [Trp 8116; Leu 9779; n.s.]. Schlussfolgerung Diese GKV-Versorgungsforschungsstudie beschreibt die Anwendung von GnRHa zur initialen Therapie beim fortgeschrittenen $\mathrm{PCa}$ in Deutschland und zeigt substanzabhängige Unterschiede in den Verordnungsmustern, Hospitalisierungsraten und Krankenhauskosten.

\section{ABSTRACT}

Introduction: Androgen deprivation therapy (ADT) plays a pivotal role in the treatment of advanced or metastasised prostate cancer (PCa). The aim of this health services research was to compare real-world data on the initial use of different $\mathrm{GnRH}$ agonists and antagonists ( $\mathrm{GnRHa}$ ) with regard to prescription patterns, hospitalisation rates and costs.

Material and methods: Anonymised claims data from $>70$ German health insurance funds between 2010 and 2015 (n $=4205227$ ) were analysed ( 1 year pre-observation period, 1 index quarter with initial GnRHa prescription, $\geq 2$ years of follow-up (FU)).

Results: The study population included 2382 PCa patients (mean age 75 years). Leuprolide (Leu) was prescribed most frequently (56.6\%). At initial GnRHa administration, $70 \%$ of patients neither had lymph node nor distant metastases. Around $11.2 \%$ of all patients stopped GnRHa treatment after the first prescription, $17.6 \%$ switched their initial therapy to another substance after a mean of 457 days (median: 399 days); in the hybrid (hyb) group 100 days earlier on average than in the agonist group $(p=0.016)$. The prevalence ranking of the most common comorbidities was consistent over time: hypertension, hyperlipidaemia, cardiovascular disease (CVD) and diabetes. The prevalence of hypertension increased significantly in the agonist group (16.4\%) compared with the antagonist $(6.9 \%, p=0.022)$ and hyb group $(11.6 \%, p=0.006)$. With regard to CVD, there were no significant differences in the relative growth rate between the 3 combined therapy classes. In total, 23.9 $\%$ of all patients died within the 3-year FU. The mortality rate was lowest for triptorelin (Trp, $22.1 \%$ ) and highest for goserelin (Gos, $29.4 \%$, n.s.). In the index quarter, $26.4 \%$ of patients had at least one inpatient hospitalisation [minmax: $\operatorname{Trp} 22.4 \%$; Gos $30.3 \%$ ], with an average length of hospital stay/patient of 3 days [Trp 2.4; Gos 4.5]. The annual hospitalisation rate was between 36.2 and $40.7 \%$, the average length of hospital stay in the entire FU was between 17.6 (Trp) and 20.8 days (hyb). The average hospital costs in the index quarter were approx. EUR 1200 [Trp 988; Gos 1803] and per FU year approx. EUR 3000. In the Trp cohort, total costs (index quarter +3 years) were more than EUR 1000 below the average costs of EUR 9476 [Trp 8116; Leu 9779; n.s.].

Conclusion: This comparative retrospective analysis provides real-world information on initial GnRHa treatment for advanced prostate cancer, revealing differences in treatment patterns, hospitalisation rates and hospital costs in Germany.

\section{Einleitung}

Das Prostatakarzinom (PCa) war mit 57370 Neuerkrankungen im Jahr 2014 die häufigste Krebserkrankung bei Männern in Deutschland und tritt v.a. im Alter auf [1]. Das PCa gehört nach wie vor zu den Krebserkrankungen mit der höchsten Mortalität [2]. Ursachen und Risikofaktoren bedürfen zwingend weiterer Klärung [3], es sind ebenfalls dringend Studien zur Optimierung der aktuellen Behandlungsrealität vonnöten [4].

Aufgrund des hormonabhängigen Wachstums des PCa ist die Testosteronsuppression zur wichtigsten Behandlungsstrategie in der palliativen Situation geworden [5]. Bei Männern mit lokal fortgeschrittenem PCa, als Begleitung zur Radiatio oder bei metastasiertem PCa ist die Hormonentzugstherapie (androgen deprivation therapy; ADT) die tragende Säule der Behandlung. Sowohl durch die chirurgische als auch medikamentöse Kastration wird die Testosteronkonzentration signifikant gesenkt (<20-50ng/dl, vorzugsweise<20 ng/dl) [6,7]. Für die medikamentöse ADT werden im Wesentlichen $\mathrm{GnRH}$ Agonisten und GnRH-Antagonisten eingesetzt. Zu den am häufigsten verwendeten $\mathrm{GnRH}$-Agonisten gehören Leuprorelin, Triptorelin, Goserelin und Buserelin [8]. Der derzeit einzige in Deutschland zugelassene GnRH-Antagonist ist Degarelix.
GnRH-Agonisten und -Antagonisten unterscheiden sich in ihrem Wirkmechanismus. GnRH-Agonisten binden an die GnRH-Rezeptoren der Hypophyse und vermitteln die gleiche Wirkung wie endogenes GnRH. Die fortwährende Verabreichung führt jedoch zu einer kontinuierlichen Stimulation und damit zu einer Herunterregulierung dieser Rezeptoren, sodass die LH- und FSH-Spiegel sinken und der Testosteronwert auf Kastrationsniveau fällt.

Im Gegensatz dazu verhindern GnRH-Antagonisten die Freisetzung von $\mathrm{LH}$ und FSH durch eine kompetitive Blockade des GnRH-Rezeptors [9]. Antiandrogene, z. B. Bicalutamid oder Flutamid, verhindern die Bindung von Testosteron am Androgenrezeptor $[10,11]$. Hormonmanipulierende Substanzen der nächsten Generation sind Enzalutamid und Abirateron, die in der metastasierten kastrationsresistenten Situation zugelassen sind und eingesetzt werden [12,13]. Abirateron wirkt auch im metastasierten hormonsensitiven PCa (in Kombination mit ADT) lebensverlängernd, sodass nun auch seit Kurzem hierfür eine Zulassung existiert [14]. Apalutamid ist ein weiteres neues nicht steroidales Antiandrogen, welches das metastasenfreie Überleben beim nicht metastasierten kastrationsresistenten PCa verbessert und nun unter anderem in Deutschland und den USA für diese Situation zugelassen wurde [15]. Auch für Enzalutamid besteht nun eine Zulassungserweiterung für das 
nicht metastasierte kastrationsresistente PCa [16] Die ADT ist auch im kastrationsresistenten Stadium eine fest etablierte Basistherapie und sollte lebenslang fortgesetzt werden [17].

Trotz der großen Bedeutung der ADT für die Behandlung des fortgeschrittenen und metastasierten PCa enthalten die bestehenden Leitlinien nur wenig Informationen zur Differenzierung von GnRH-Agonisten und -Antagonisten. Das Ziel der vorliegenden Studie war es daher, den Behandlungsalltag in Deutschland hinsichtlich Verordnungsverhalten und -mustern, Hospitalisierungen und Krankenhauskosten in den ersten 3 Jahren nach Beginn der Behandlung zu beschreiben und je nach GnRHaSubstanzklasse auszuwerten. Folgende Wirkstoffe und Substanzklassen wurden berücksichtigt: die Agonisten Buserelin, Goserelin, Leuprorelin, Triptorelin, die Leuprorelin-Hybride ${ }^{1}$ und der Antagonist Degarelix. Die Ergebnisse werden im Folgenden in 4 Abschnitten dargestellt: 1) Patientencharakteristika, 2) Komorbidität und Mortalität, 3) Therapiemuster sowie 4) Hospitalisierung. Die ersten Ergebnisse mit Fokus auf den Krankheitsverlauf sind in einer kürzlich erschienenen Studie unserer Arbeitsgruppe ausführlicher beschrieben [18]. Bei der vorliegenden Studie mit Schwerpunkt Gesundheitsökonomie liegt der Fokus hingegen auf den Therapie- und Verschreibungsmodalitäten sowie den Krankenhaustagen und -kosten.

\section{Material und Methoden}

Es wurde eine retrospektive Versorgungsforschungsanalyse von Routinedaten der gesetzlichen Krankenkassen (GKV) in Deutschland durchgeführt.

\section{Datenquelle}

Anonymisierte Daten von $>70$ deutschen GKV, v.a. Betriebskrankenkassen (BKK) und Innungskrankenkassen (IKK), wurden ausgewertet [19]. Die Kohorte umfasste GKV-versicherte Patienten zwischen 2010 und 2015.

\section{Studienpopulation}

Die Ein- und Ausschlusskriterien der Studienpopulation sind in - Abb. 1a dargestellt. Einige ausgewählte Fragen wurden mit einer größeren Population untersucht, die im Folgenden als „adjustierte Population“ bezeichnet wird. Die von der Studienpopulation abweichenden Auswahlkriterien der adjustierten Population sind ebenfalls in $>$ Abb. 1 a enthalten.

\section{Studiendauer}

Eingeschlossen wurden Patienten, die zwischen 1.Januar 2010 und 31. Dezember 2015 mindestens einen Tag versichert waren. Das Akquisitionsintervall für die erste beobachtete Behandlung mit GnRHa umfasste den Zeitraum vom 1. Januar 2011 bis zum 31. Dezember 2012, um eine GnRHa-freie Prä-Indexperiode von einem Jahr (4 Quartale zwischen dem 1.Januar 2010 und dem 31. Dezember 2010) und eine Follow-Up-Peri-

\footnotetext{
${ }^{1}$ In Deutschland besaßen zum Zeitpunkt der Analyse (2017) 2 GnRH-Produkte eine sog. Hybrid-Zulassung. Eine Hybrid-Zulassung ist eine Zulassung. die sich auf ein Originalprodukt bezieht; die Substanz erfüllt jedoch nicht alle Kriterien für ein Generikum, sodass zusätzliche präklinische und klinische Daten vorgelegt werden müssen.
}

\section{a Eingeschlossene Patienten}

\begin{tabular}{|c|}
\hline $\begin{array}{l}\text { Alle GKV-Versicherten, die zwischen } 2010 \text { und } 2015 \text { mindestens } \\
\text { einen Tag versichert waren }(\mathrm{N}=4.205 .227)\end{array}$ \\
\hline$\longrightarrow$ Ausgeschlossen: $\mathrm{N}=2.146 .030$ \\
\hline $\begin{array}{l}\text { Alle männlichen GKV-Versicherten, die zwischen } 2010 \text { und } 2015 \\
\text { mindestens einen Tag versichert waren }(\mathrm{N}=2.059 .197)\end{array}$ \\
\hline$\rightarrow$ Ausgeschlossen: $\mathrm{N}=336.426$ \\
\hline $\begin{array}{l}\text { Alle männlichen GKV-Versicherten, die zwischen } 2010 \text { und } 2015 \\
\text { oder bis zu ihrem Tod kontinuierlich versichert waren }(\mathrm{N}=1.722 .771)\end{array}$ \\
\hline$\rightarrow$ Ausgeschlossen: $\mathrm{N}=1.666 .267$ \\
\hline $\begin{array}{l}\text { Alle männlichen GKV-Versicherten mit Prostatakarzinom- } \\
\text { Diagnose zwischen } 2010 \text { und } 2015(\mathrm{~N}=56.504)\end{array}$ \\
\hline$\rightarrow$ Ausgeschlossen: $\mathrm{N}=19.328$ \\
\hline $\begin{array}{l}\text { Alle männlichen GKV-Versicherten ohne einen anderen primären } \\
\text { Tumor zwischen } 2010 \text { und } 2015(\mathrm{~N}=37.176)\end{array}$ \\
\hline$\rightarrow$ Ausgeschlossen: $\mathrm{N}=27.151$ \\
\hline $\begin{array}{l}\text { Alle männlichen GKV-Versicherten mit einer Therapie mit GnRH- } \\
\text { Agonisten und -Antagonisten zwischen } 2010 \text { und } 2015(\mathrm{~N}=10.025)\end{array}$ \\
\hline$\rightarrow$ Ausgeschlossen: $\mathrm{N}=11$ \\
\hline $\begin{array}{l}\text { Alle männlichen GKV-Versicherten mit einer Therapie mit GnRH- } \\
\text { Agonisten und -Antagonisten sowie Prostatakarzinomdiagnose } \\
\text { im selben Jahr zwischen } 2010 \text { und } 2015(\mathrm{~N}=10.014)\end{array}$ \\
\hline$\longrightarrow$ Ausgeschlossen: $\mathrm{N}=3.822(2.872)$ \\
\hline $\begin{array}{l}\text { Alle männlichen GKV-Versicherten mit einer Therapie } \\
\text { mit GnRH-Agonisten und GnRH-Antagonisten sowie } \\
\text { Prostatakarzinomdiagnose im selben Jahr zwischen } 2011 \text { und } \\
2012 \text { (2011 und 2013) }(\mathrm{N}=6.192(7.142))\end{array}$ \\
\hline$\rightarrow$ Ausgeschlossen: $\mathrm{N}=2(2)$ \\
\hline $\begin{array}{l}\text { Alle männlichen Versicherten mit einer Verschreibung von } \\
\text { entweder GnRH-Agonist oder -Antagonist am ersten Tag der } \\
\text { Verschreibung (Indexdatum) zwischen } 2011 \text { und } 2012 \\
\text { (2011 und 2013) }(\mathrm{N}=6.190 \text { (7.140)) }\end{array}$ \\
\hline$\rightarrow$ Ausgeschlossen: $\mathrm{N}=3.808(3.419)$ \\
\hline $\begin{array}{l}\text { Alle männlichen GKV-Versicherten ohne GnRH-Agonisten und } \\
\text {-Antagonisten in den ersten } 4 \text { (2) Quartalen vor ihrer ersten } \\
\text { Verschreibung ( } \mathrm{N}=2.381 \text { (3.721)) }\end{array}$ \\
\hline
\end{tabular}

\section{b Studiendesign}

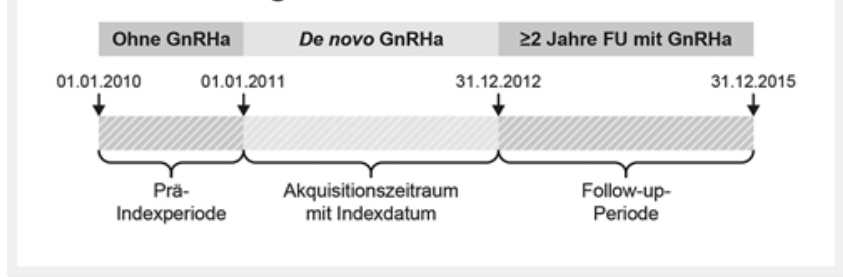

- Abb. 1 a Auswahl der Studienpopulation. Kursiv: Adjustierte Population. b Studiendesign.

ode (FU) von 3 Jahren (zwischen 1. Januar 2013 und 31. Dezember 2015) zu erhalten ( $\triangleright$ Abb. 1b). Die Prä-indexperiode betrug ein Jahr, um möglichst nur Patienten einzuschließen, bei denen eine ADT initiiert wurde; durch eine kürzere Präindexperiode wären auch Patienten in Therapiepausen/-unterbrechungen eingeschlossen worden. Der zeitliche Rahmen für den individu- 
ellen Studienzeitraum jedes einzelnen Patienten wurde dabei durch das Indexdatum, d.h. das jeweilige initiale GnRHa-Verschreibungsdatum innerhalb des Akquisitionszeitraums (2011/ 2012), definiert.

Für eine erweiterte Komorbiditätenanalyse wurde die Patientenkohorte durch Adjustierung der Selektionskriterien vergrößert. Die höhere Zahl der Patienten in der adjustierten Population ( $n=3721$ gegenüber $n=2382$ ) resultierte aus einer längeren Indexperiode (2011 bis 2013) und einer kürzeren Prä-Indexperiode (2 Quartale) im Vergleich zur Studienpopulation ( Abb. 1a). Das FU der adjustierten Population war mit 2 Jahren kürzer als das der Studienpopulation.

\section{Patientenkohorten}

Die Studienpopulation wurde in 6 Patientenkohorten unterteilt, die auf der ersten Verschreibung der folgenden $6 \mathrm{GnRHa-}$ Substanzen basierten: Buserelin, Goserelin, Leuprorelin (ohne Leuprorelin-Hybride), Triptorelin, Leuprorelin-Hybride und Degarelix.

\section{Behandlungsmuster}

Folgende Behandlungsmuster wurden ausgewertet: Wechsel, Fortführung, Abbruch, Unterbrechung und Therapiephase $(\triangleright$ Tab. 1).

\section{Statistik}

Die Ergebnisse wurden mittels deskriptiver Statistik ausgewertet. Für die statistische Analyse wurden der Chi-Quadrat-Test (kategoriale Variablen) und der Wilcoxon-Rank-Summentest (kontinuierliche Variablen) angewandt. Ein p-Wert von<0,05 wurde als statistisch signifikant definiert.

\section{Ergebnisse}

\section{Patientencharakteristika}

Insgesamt wurden 2382 Patienten in die Analyse mit der IndexGnRHa-Therapie 2011/2012 (4 Quartale Prä-Indexperiode) eingeschlossen ( $\boldsymbol{A} \mathbf{b} \mathbf{b} \mathbf{b} \mathbf{1 a}$ ) und entsprechend ihrer initialen GnRHa-Verordnung in 6 Hauptkohorten unterteilt ( $\mathbf{A b b . 2 ) .}$ Die Patientencharakteristika sind in $>$ Tab. 2 dargestellt. Das Durchschnittsalter der Patienten betrug 75 Jahre. Die jährlichen Prävalenzen lagen zwischen 2,0 und 2,3\% (2010-2014); die Altersgruppe der 80 -bis 89 -Jährigen wies die höchsten jährlichen Prävalenzraten (ca. 12\%) auf. Bei der ersten GnRHaBehandlung zeigten $70 \%$ der Patienten keine Lymphknotenoder Fernmetastasen. Vorweg genommen ist bei der Interpretation dieser hohen Zahl der Ursprung der Daten (Krankenversicherungsdaten zum Zweck der Abrechnung) und damit verbunden eine mögliche Kodierungsungenauigkeit bzw. Unterschätzung der Metastasenprävalenz (im Vergleich zu klinischen Daten) zu beachten. Die Patientengruppe, die initial Degarelix erhielt, war am jüngsten (mittleres Alter 72 Jahre) und wies die höchste Rate an Lymphknoten- und Fernmetastasen auf (38\%; vs. $\operatorname{Trp} 19 \% \mathrm{p}=0,002)$.
- Tab. 1 Definitionen der Behandlungsmuster.

\begin{tabular}{|c|c|}
\hline $\begin{array}{l}\text { Behandlungs- } \\
\text { muster }\end{array}$ & Definition \\
\hline Wechsel & Wechsel zu einem anderen Wirkstoff im FU* \\
\hline Fortführung & Weitere Verschreibung eines Wirkstoffs \\
\hline Abbruch & $\begin{array}{l}\text { Zeitraum von> } 6 \text { Monaten vom Datum der letz- } \\
\text { ten Verschreibung bis zum Ende des FU ohne } \\
\text { weitere Verschreibung oder Tod eines Patien- } \\
\text { ten im FU. Das Datum des Therapieabbruchs } \\
\text { wurde als Todestag oder } 6 \text { Monate nach dem } \\
\text { letzten Verordnungsdatum definiert. }\end{array}$ \\
\hline Unterbrechung & $\begin{array}{l}\text { Zeitraum von }>6 \text { Monaten zwischen } 2 \text { Ver- } \\
\text { schreibungen. Das Datum der Therapieunter- } \\
\text { brechung wurde definiert als der erste Tag } 6 \\
\text { Monate nach der letzten Verschreibung. Die } \\
\text { Dauer der Therapieunterbrechung wurde aus } \\
\text { dem ersten Tag des Therapieabbruchs und dem } \\
\text { Datum der nächsten Medikamentenverord- } \\
\text { nung berechnet. }\end{array}$ \\
\hline $\begin{array}{l}\text { Therapie-/ } \\
\text { Behandlungs- } \\
\text { phase }\end{array}$ & $\begin{array}{l}\text { Beginn: erste Verschreibung eines Wirkstoffs } \\
\text { Ende: Wechsel, Abbruch oder Unterbrechung } \\
\text { dieser Wirkstoffverordnung }\end{array}$ \\
\hline
\end{tabular}

\section{Patienten pro Indextherapie (Kohorten) in den Indexjahren 2011/12}

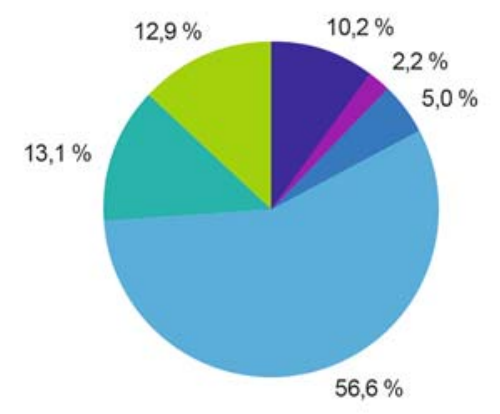

- Buserelin

- Degarelix

- Goserelin

= Leuprorelin

= Leuprorelin-Hybride

in Triptorelin

Abb. 2 Anteil der Patienten pro Indextherapie (Kohorten) in den Indexjahren 2011/12.

\section{Komorbidität und Mortalität}

Die häufigsten Komorbiditäten zum Zeitpunkt der GnRHa-Initiierung und auch im FU waren Hypertonie (71,5\%; FU: 85,0\%), gefolgt von Hyperlipidämie (45,2\%; 60,8\%), kardiovaskulären Erkrankungen (35,7\%; 54,1\%) und Diabetes mellitus (28,3\%, $36,2 \%)$. Die seltenste Komorbidität waren sonstige Knochenkrankheiten $(0,8 \% ; 3,1 \%)$ ( $\mathbf{A b b}$. 3 ). Die beobachteten Komorbiditätsprävalenzen waren vergleichbar mit den 5-Jahres-Prävalenzraten der Benchmarkpopulation „Alle männlichen GKVVersicherten mit Prostatakarzinomdiagnose zwischen 2010 und 2015 ohne weiteren Primärtumor“ ( $n=37220)$. Da aus der Studienpopulation keine statistisch signifikanten Unterschiede hinsichtlich der Komorbiditätsraten im Vergleich zwischen den 
- Tab.2 Demografische Daten der Studienpopulation ( $\mathrm{n}=2382$ ).

\begin{tabular}{|c|c|c|c|c|c|c|c|}
\hline & \multirow[b]{2}{*}{$\begin{array}{l}\text { Gesamt- } \\
\text { population } \\
(\mathrm{n}=\mathbf{2 3 8 2})\end{array}$} & \multicolumn{6}{|l|}{ Kohorten } \\
\hline & & $\begin{array}{l}\text { Buserelin } \\
(n=244)\end{array}$ & $\begin{array}{l}\text { Degarelix } \\
(n=52)\end{array}$ & $\begin{array}{l}\text { Goserelin } \\
(n=119)\end{array}$ & $\begin{array}{l}\text { Leuprorelin } \\
(\mathrm{n}=1.347)\end{array}$ & $\begin{array}{l}\text { Leuprorelin- } \\
\text { Hybride } \\
(\mathrm{n}=312)\end{array}$ & $\begin{array}{l}\text { Triptorelin } \\
(\mathbf{n}=\mathbf{3 0 8})\end{array}$ \\
\hline $\begin{array}{l}\text { Mittleres Alter, } \\
\text { Jahre, (Median) }\end{array}$ & $\begin{array}{l}75 \\
(75)\end{array}$ & $\begin{array}{l}75 \\
(76)\end{array}$ & $\begin{array}{l}72 \\
(74)\end{array}$ & $\begin{array}{l}75 \\
(75)\end{array}$ & $\begin{array}{l}75 \\
(75)\end{array}$ & $\begin{array}{l}76 \\
(76)\end{array}$ & $\begin{array}{l}75 \\
(75)\end{array}$ \\
\hline \multicolumn{8}{|l|}{ Altersgruppe (\%) } \\
\hline $0-39$ & 0,00 & 0,00 & 0,00 & 0,00 & 0,00 & 0,00 & 0,00 \\
\hline $40-49$ & 0,08 & 1,02 & 0,00 & 0,00 & 0,07 & 0,00 & 0,00 \\
\hline $50-59$ & 3,61 & 2,46 & 11,54 & 2,10 & 4,01 & 3,53 & 1,62 \\
\hline $60-69$ & 17,63 & 13,93 & 19,23 & 22,69 & 17,89 & 15,06 & 19,81 \\
\hline $70-79$ & 50,13 & 58,20 & 51,92 & 41,18 & 49,15 & 47,76 & 53,57 \\
\hline $80-89$ & 26,66 & 23,36 & 15,38 & 31,93 & 26,80 & 31,41 & 23,70 \\
\hline $90+$ & 1,89 & 1,02 & 1,92 & 2,10 & 2,08 & 2,24 & 1,30 \\
\hline \multicolumn{8}{|c|}{ PCa-Stadium * (\%) } \\
\hline Gesamt & 100 & 100 & 100 & 100 & 100 & 100 & 100 \\
\hline NO MO & 70,07 & 68,85 & 50,00 & 63,87 & 71,05 & 70,51 & 72,08 \\
\hline N1 M0 & 3,90 & 3,69 & 1,92 & 0,84 & 4,31 & 3,85 & 3,90 \\
\hline N0 M1 & 6,51 & 4,92 & 9,62 & 10,08 & 6,83 & 6,09 & 4,87 \\
\hline N1 M1 & 19,52 & 22,54 & 38,46 & 25,21 & 17,82 & 19,55 & 19,16 \\
\hline
\end{tabular}



- Abb. 3 Auftreten von Komorbiditäten im Indexquartal (unterer Teil des Balkens) und Follow-Up (oberer Teil des Balkens). verwendeten Therapien hervorgingen, wurde eine adjustierte Population herangezogen; diese umfasste 3721 Patienten. Die häufigsten Komorbiditäten der adjustierten Population entsprachen denen der Studienpopulation. Zwischen den GnRHAgonisten $(n=3149)$ und dem $\mathrm{GnRH}$-Antagonisten $(n=81)$ wurden signifikante Unterschiede hinsichtlich des relativen Anstiegs der Hypertonie- und der obstruktiven Uropathie-Prävalenz während des FU beobachtet: Während unter GnRH-Agonisten die Hypertonie signifikant deutlicher zunahm (16,4\% vs. 6,9\% Antagonisten; $p=0,022$ ), wurde unter Therapie mit Antagonisten eine signifikant höhere Zunahme der obstruktiven Uropathie beobachtet (100,0\% vs. 70,1\% Agonisten; $p<0,001$ ). Auch zwischen GnRH-Agonisten und Hybriden $(n=491)$ gab es signifikante Unterschiede in der Prävalenzzunahme von Hypertonie (16,4\% für GnRH-Agonisten vs. 11,6\% für Hybride; $p=$ 0,006) und obstruktiver Uropathie (70,1\% für GnRH-Agonisten vs. $100 \%$ für Hybride; $p<0,001)$. Obwohl signifikante Unterschiede in Bezug auf Hypertonie beobachtet wurden, konnten keine signifikanten Unterschiede hinsichtlich kardiovaskulärer Erkrankungen und Diabetes festgestellt werden.

Fast ein Viertel $(23,9 \%, n=570)$ aller PCa-Patienten verstarb während des FU. In der Triptorelin-Kohorte wurde mit 22,1\% die niedrigste Mortalitätsrate $(n=68)$ beobachtet, während die Goserelin-Kohorte mit 29,4\% $(n=35)$ die höchste Mortalitätsrate aufwies (n.s.; $>$ Tab. 3 ). 
Tab. 3 Mittlere und mediane Tage bis zum Tod (FU) und Mortalitätsrate (Indexquartal und FU).

\begin{tabular}{|c|c|c|c|c|}
\hline \multirow[b]{2}{*}{ Therapiekohorte } & \multicolumn{3}{|c|}{ Tage bis zum Tod } & \multirow{2}{*}{$\begin{array}{l}\text { Mortalitätsrate } \\
(p=\text { n.s. }) \\
\%\end{array}$} \\
\hline & $\mathbf{N}$ & Mittelwert & Median & \\
\hline Alle & 570 & 534,1 & 522,0 & 23,93 \\
\hline $\begin{array}{l}\text { Buserelin } \\
(n=244)\end{array}$ & 54 & 579,4 & 592,5 & 22,13 \\
\hline $\begin{array}{l}\text { Goserelin } \\
(n=119)\end{array}$ & 35 & 515,6 & 535,0 & 29,41 \\
\hline $\begin{array}{l}\text { Leuprorelin } \\
(n=1.347)\end{array}$ & 315 & 527,9 & 517,0 & 23,39 \\
\hline $\begin{array}{l}\text { Leuprorelinhybride } \\
(\mathrm{n}=312)\end{array}$ & 83 & 535,1 & 535,0 & 26,60 \\
\hline $\begin{array}{l}\text { Triptorelin } \\
(n=308)\end{array}$ & 68 & 523,9 & 477,5 & 22,08 \\
\hline $\begin{array}{l}\text { Degarelix } \\
(n=52)\end{array}$ & 15 & 584,6 & 534,0 & 28,85 \\
\hline
\end{tabular}

\section{a Anteil an Patienten mit Wechsel nach Indextherapie}



\section{b Durchschnittliche Tage bis zum ersten Wechsel nach Indextherapie (Therapieklassen)}



- Abb. 4 Ergebnisse zu Therapiewechsel. a Anteil an Patienten mit Wechsel nach der Indextherapie, ${ }^{*} \mathrm{p}<0,001$ vs. Triptorelin. b Mittlere Anzahl der Tage bis zum 1. Wechsel nach Indextherapie basierend auf Therapieklassen, ${ }^{*} p<0,05$ vs. Agonisten.

\section{Therapiemuster}

Therapiewechsel, -abbruch und -unterbrechung

Insgesamt wechselten $17,6 \%$ aller Patienten der Studienpopulation die GnRHa-Therapie innerhalb von 3 Jahren. Die höchsten Wechsel-Raten wurden bei Degarelix und Goserelin festgestellt und waren signifikant höher als bei Triptorelin $(p<$ 0,001 ); in der Triptorelin-Kohorte war die Wechsel-Rate wiederum signifikant höher als in der Leuprorelin Index-Gruppe $(p<$ 0,001) ( Abb.4a). In zusammengefassten Therapieklassen kam es bei den Hybriden mit durchschnittlich 100 Tagen signifikant schneller zu einem Wechsel als bei den GnRH-Agonisten (377 vs. 476 Tage, $p=0,016$ ) ( $\triangleright$ Abb. 4b).
Der erste Wechsel war in allen Studiengruppen am häufigsten zu Leuprorelin (>50\%), in der Leuprorelin-Gruppe am häufigsten zu Hybriden und Triptorelin (jeweils 30\%). Insgesamt wurde am häufigsten zu Leuprorelin (36,8\%) gewechselt, gefolgt von Triptorelin (18,1\%), Buserelin (17,2\%) und Hybriden $(15,6 \%)$. Bis zum ersten Wechsel vergingen im Durchschnitt 457 Tage (Median: 398,5 Tage; min. Hyb 308, max. Gos 475). Bei Patienten, die initial mit Leuprorelin-Hybriden behandelt wurden, kam es im Mittel signifikant schneller zu einem Wechsel als bei Patienten, die initial mit Triptorelin (377 vs. 495 Tage; $\mathrm{p}=0,025$ ) behandelt wurden.

Nach der initialen Verordnung wurden bei 11,2\% die GnRHa abgesetzt, über die Hälfte (58,2\%) der Patienten der Studien- 
population, die mit einer GnRHa-Therapie begannen, brachen ihre Therapie innerhalb von 3 Jahren ab-im Durchschnitt nach 527 Tagen (inklusive Tod). Es gab keine signifikanten Unterschiede hinsichtlich der Abbruchraten zwischen den 6 Indextherapie-Kohorten. Die meisten Abbrüche erfolgten nach Leuprorelin (56,0\%), gefolgt von Abbrüchen nach Triptorelin (14,4 $\%)$, Leuprorelin-Hybriden (13,0\%) und Buserelin (10,8\%). Fast $40 \%$ der Patienten unterbrachen die Therapie. Zwischen Triptorelin (38,6\%) und den anderen Therapiegruppen-außer der Hybridkohorte $(27,9 \%, p=0,004)$-gab es keine signifikanten Unterschiede hinsichtlich der Patientenzahl mit mindestens einer Therapieunterbrechung. In der gesamten Studienpopulation wurde die Therapie innerhalb der ersten 3 Jahre im Mittel 1,6-mal unterbrochen; die mittlere Länge einer Therapiepause betrug 139 Tage. Auf Verordnungen nach Leuprorelin entfiel mit 63,8\% der höchste Anteil aller Therapiepausen innerhalb des FU.

\section{Zusätzliche Therapien}

Innerhalb von 3 Jahren nach GnRHa-Therapieinitiierung wurde bei $14,3 \%$ der Studienpopulation eine Therapie mit Antiandrogenen begonnen, 6,5\% erhielten eine initiale Therapie mit Abirateron $^{2}$, bei 5,2\% wurde eine Strahlentherapie initiiert, ebenfalls $5,2 \%$ begannen eine Chemotherapie mit Docetaxel ${ }^{3}$, bei 2,9\% wurde eine Prostatektomie durchgeführt und 1,9\% bekamen Enzalutamid ${ }^{4}$. Andere Therapien wie die Orchiektomie, eine Chemotherapie mit Cabazitaxel ${ }^{5}$, eine Radionuklidtherapie oder eine Becken-Lymphadenektomie wurden nur selten oder gar nicht durchgeführt. Die Abbruchraten der GnRHa-Therapie (inkl. Tod) bei Beginn einer zusätzlichen medikamentösen Therapie rangierte zwischen 18,1\% (Abirateron), 20,2\% (Docetaxel) und 24,4\% (Enzalutamid).

\section{Verschreibungsverhalten}

Die erste Therapiephase dauerte durchschnittlich 476 Tage. In dieser Phase erfolgten 3,7 GnRHa-Verschreibungen pro Patienten. Es wurde kein Unterschied in Bezug auf die der verschiedenen GnRHa-Verschreibungen durch die unterschiedlichen Facharztgruppen festgestellt: Über $90 \%$ der Verordnungen wurden, wie zu erwarten, von Urologen getätigt. Lediglich bei Goserelin verschob sich die Verschreibungsrate etwas zu den Onkologen mit $12 \%$ vs. $80 \%$ Urologen vs. Andere ( $\mathbf{A b b}$. 5). Bei der Analyse der genannten Therapiemuster resultiert ein identisches Bild: $90 \%$ aller Verordnungen wurden bei jeder Art von Therapiephase (z. B. vor Wechsel, Pause, Abbruch, etc.) vom Urologen getätigt.

\section{Hospitalisierung}

Insgesamt 26,4\% der Studienpopulation hatte mindestens einen stationären Aufenthalt im Indexquartal. Die geringste Hospitalisierungsrate wies die Triptorelin-Kohorte (22,4\%) auf. Die jährliche Hospitalisierungsrate (nach Indexquartal) betrug zwi-

\footnotetext{
2 ATC-Code: L02BX03, Marktzulassung in Deutschland 2011

3 ATC-Code: L01CD02, Marktzulassung in Deutschland 2004

${ }^{4}$ ATC-Code: L02BB04, Marktzulassung in Deutschland 2013

5 ATC-Code: L01CD04, Marktzulassung in Deutschland 2011
}

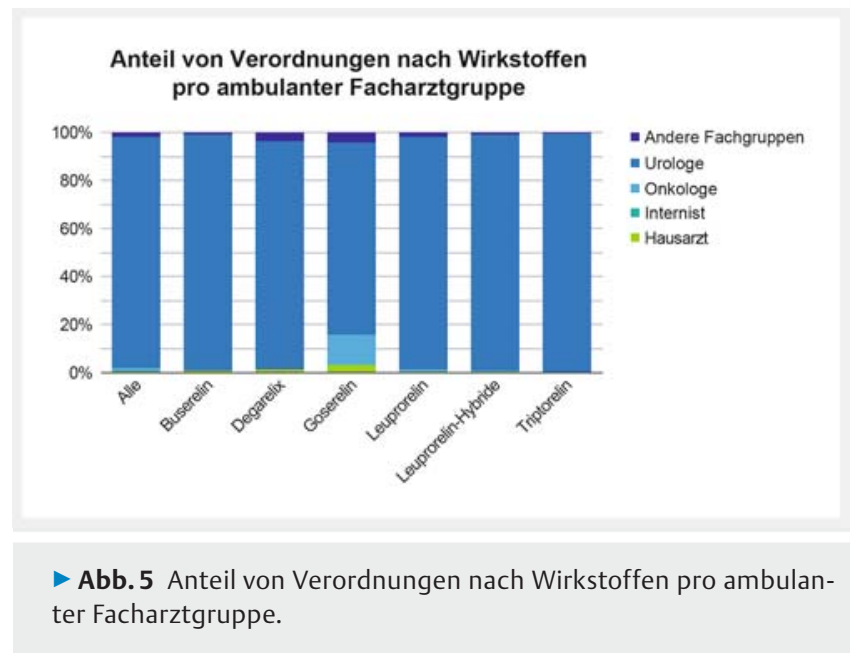

schen 36,2\% und 40,7\%. Die mittlere Anzahl der Krankenhausaufenthalte unter den Risikopatienten (Ausschluss verstorbener Patienten) betrug 0,4 im Indexquartal, im ersten Jahr des FU 0,8 und in den Jahren 2 und 3 jeweils 0,7. In der DegarelixKohorte war die mittlere Anzahl der Hospitalisierungsfälle mit 3,3 über das gesamte FU signifikant höher $(p=0,033)$ als bei Triptorelin (2,2) ( $\mathbf{A b b}$.6a). Die mittlere Hospitalisierungsdauer pro Patient betrug 3,0 Tage im Indexquartal und 20,1 Tage im gesamten Beobachtungszeitraum. Die Triptorelin-Kohorte wies mit 2,4 Tagen im Indexquartal und 17,6 Tagen im gesamten FU die kürzeste Hospitalisierungsdauer auf (n.s.).

Der mittlere Betrag der stationären Kosten für die Studienpopulation im Indexquartal lag bei etwa 1200 Euro; die niedrigsten durchschnittlichen Kosten pro Patient im Krankenhaus wurden im Index-Quartal in der Triptorelin-Kohorte (987 Euro) festgestellt, die höchsten Kosten für Goserelin (1803 Euro). In jedem FU-Jahr betrugen die durchschnittlichen Krankenhausaufenthaltskosten etwa 3000 Euro. Bei der Benchmarkpopulation „Alle männlichen GKV-Versicherten mit Prostatakarzinomdiagnose zwischen 2010 und 2015 ohne weiteren Primärtumor" ( $n=37220)$ bewegten sich die jährlichen Krankenhauskosten im Zeitraum 2010-2015 zwischen ca. 1800 und 2500 Euro. Nach dem ersten FU-Jahr reduzierten sich die Kosten in der Triptorelin-Gruppe (3132 Euro in Jahr 1, 2233 Euro in Jahr 2 und 2344 Euro in Jahr 3) im Vergleich zu den anderen Kohorten und lagen dann mit 8116 Euro im Gesamtbeobachtungszeitraum etwa 1000 Euro unter dem Durchschnitt von 9476 Euro (n.s.) ( Abb.6b).

\section{Diskussion}

Ziel dieser Versorgungsforschungsstudie war es, den Behandlungsalltag mit GnRHa zur Therapie von Patienten mit fortgeschrittenem oder metastasiertem PCa zu beschreiben und vergleichend zu analysieren.

Bei der Interpretation der Ergebnisse und der folgenden Diskussion ist jedoch zu beachten, dass die Analyse auf Daten der deutschen gesetzlichen Krankenversicherung (Sekundärdaten) beruht, deren originäre Funktion in der Abrechnung und Kostenerstattung liegt. Weiterhin ist durch den retrospektiven 


\section{a Mittlere Anzahl der Hospitalisierungsfälle}

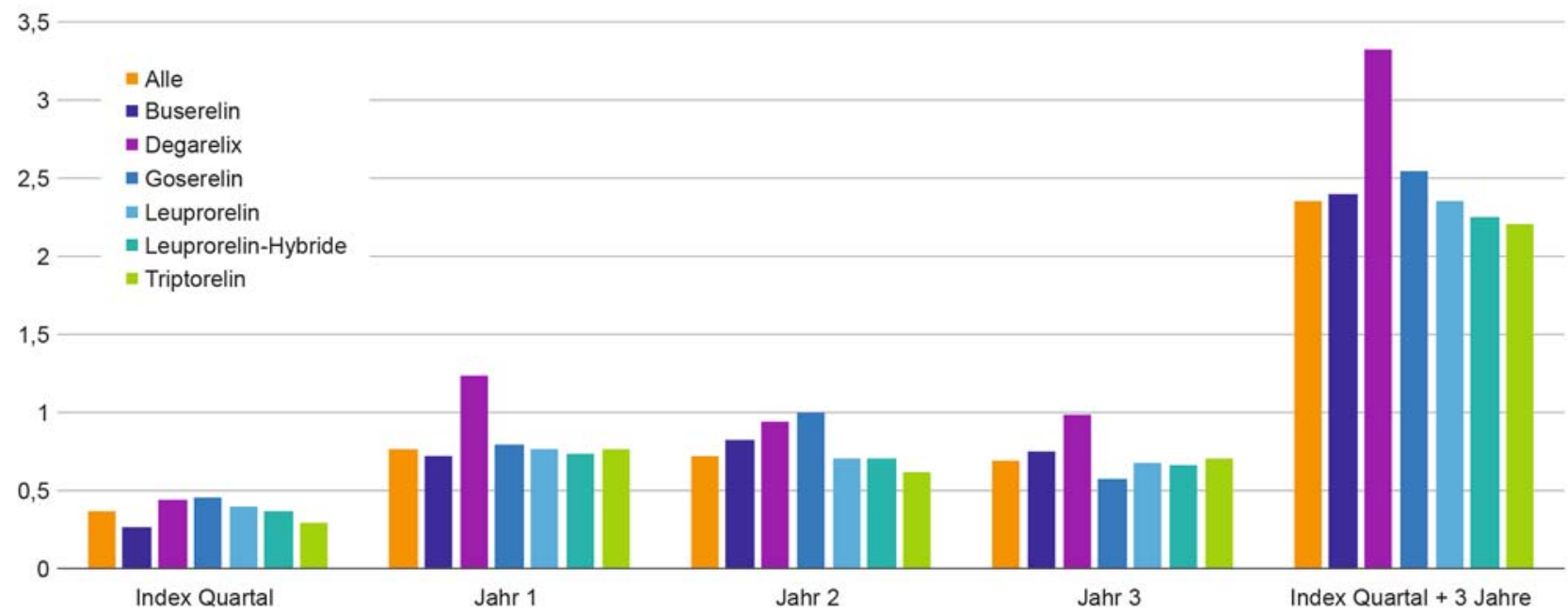

b Mittlere stationäre Behandlungskosten

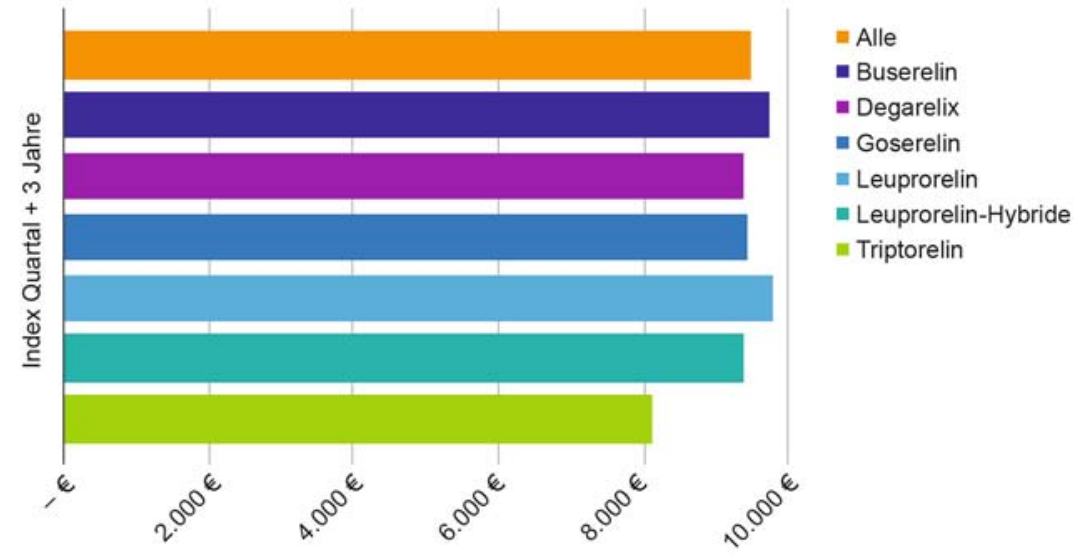

- Abb. 6 a Mittlere Anzahl der Hospitalisierungsfälle/Patient. b Mittlere stationäre Behandlungskosten.

Charakter der Studie die Dokumentationsgenauigkeit der kodierenden Arztpraxen beispielsweise in Bezug auf Komorbiditäten und Metastasen nicht zu eruieren. Die Studie unterlag daher den folgenden Limitationen: Jede Diagnose, die für die Erstattung irrelevant war, wurde möglicherweise nicht kodiert; dieses kann dazu führen, dass bestimmte klinische Ereignisse unterrepräsentiert sind oder die Prävalenz von Erkrankungen unterschätzt wird. Außerdem schließt die Verwendung von Krankenkassendaten klinische Informationen wie z. B. BMI, Raucherstatus, Laborbefunde oder Krebsrisikoklassen und patientenindividuelle Endpunkte aus. Darüber hinaus hat die Analyse möglicherweise klinisch relevante, jedoch unterhalb der statistisch signifikanten Schwelle liegende Unterschiede festgestellt, insbesondere in Bezug auf die Behandlungen mit Agonisten vs. Antagonisten in unterschiedlich großen Patientenkohorten.

Die Analyse zeigte, dass ein Großteil der Patienten bemerkenswerterweise weder Lymphknoten- noch Fernmetastasen aufwies. Möglicherweise lag hier jedoch zum Teil ein „high risk“-PCa mit temporärer Begleit-ADT zu einer Radiatio vor, welches aufgrund der fehlenden klinischen Daten in der vorliegenden Analyse nicht abgebildet werden konnte. Auch die Versorgungsforschungsstudie CAPRIS in Deutschland berichtet über einen ähnlich hohen Anteil an Patienten ohne Lymphknoten- oder Fernmetastasen und stellte bei einem Großteil dieser Patienten ein „high risk“-PCa nach den d'Amico-Kriterien fest [20]. Erneut sei hier auf die mögliche Kodierungslücke im Hinblick auf die Metastasenprävalenz hingewiesen.

In dieser Studie war Hypertonie die häufigste Komorbidität. Auch wenn keine signifikanten Unterschiede in den Anstiegsraten (Index-FU) in Bezug auf kardiovaskuläre Erkrankungen zwischen GnRH-Agonisten, -Antagonisten und Hybriden nachgewiesen wurden, wird dennoch kontrovers diskutiert, ob GnRHAgonisten zu einer höheren Inzidenz kardiovaskulärer Ereignisse führen als $\mathrm{GnRH}$-Antagonisten [21 - 26]. Aufgrund der gerin- 
gen Patientenzahl in der hier untersuchten Degarelix-Kohorte und der erhobenen Daten kann jedoch keine verlässliche Aussage getroffen werden. Eine landesweite Versorgungsforschungsstudie in Frankreich mit fast 30000 Patienten (wovon 24846 einen Agonisten und 1273 einen Antagonisten erhielten) konnte ebenfalls keinen signifikanten Zusammenhang zwischen dem Typ der GnRHa-Therapie und der Inzidenz für einen Myokardinfarkt oder ischämischen Schlaganfall aufzeigen (adjustierte HR: 1,2; $95 \%-\mathrm{KI}: 0,7-2,1)$ [27].

Nach der initialen GnRHa-Verordnung erhielten rund $11 \%$ der Patienten keine weitere GnRHa-Verordnung. Diese Zahl deckt sich in etwa mit dem Anteil der Patienten mit metastasiertem PCa, die nicht auf eine ADT ansprechen (ca. 15\%) [28]. Der Grund für ausbleibende Folgeverordnungen könnte einerseits ein Nichtansprechen auf die Behandlung mit GnRHa sein oder andererseits eine entweder nur kurzzeitig durchgeführte neoadjuvante Therapie vor Prostatektomie oder eine ebenfalls nur kurzfristig durchgeführte begleitende ADT zur Radiatio. In der vorliegenden Datenanalyse wechselten Patienten unter Leuprorelin-Hybriden das initiale Präparat signifikant schneller (im Mittel 100 Tage) als Patienten unter Agonisten und insbesondere im Vergleich zu Triptorelin (im Mittel 118 Tage). Aufgrund fehlender klinischer Parameter in diesen GKV-Daten ist nicht bekannt, ob dieser signifikant schnellere Therapiewechsel auf Compliance-Unterschiede (z. B. unterschiedliche Injektionsintervalle), lokale Nebenwirkungen (Rötungen/Indurationen der Einstichstelle) oder auf einen Progress der Erkrankung zurückzuführen ist. Zusätzlich zur GnRHa-Therapie verabreichte Therapien beinhalteten auch die neuen Substanzen Enzalutamid und Abirateron, deren Zulassungsindikation zum Studienzeitpunkt noch alleinig das kastrationsresistente Stadium betraf. Entgegen der Zulassungsstudien wurde bei etwa $20 \%$ der Patienten mit Enzalutamid oder Abirateron die ADT mit GnRHa nicht fortgeführt (inklusive Tod). Laut Empfehlungen sollte auch beim Progress in ein kastrationsresistentes Stadium die ADT fortgeführt werden [16]. Mögliche Gründe sind einerseits der Patientenwunsch oder andererseits die Einschätzung des Verordners konträr zur objektiven Datenlage.

Die Triptorelin-Gruppe wies die niedrigste Mortalitätsrate aller untersuchten Kohorten auf, der Unterschied war jedoch nicht statistisch signifikant. Diese Tendenz spiegelt sich auch in einer anderen direkten Vergleichsstudie mit 284 Patienten wider: Die 9-Monats-Überlebensrate war für Triptorelin signifikant höher als für Leuprorelin (97,0\% vs. 90,5\%; p=0,033) [29]. Auch eine Netzwerkanalyse von Uttley et al. zeigte eine niedrigere Mortalität unter Triptorelin im Vergleich zu Degarelix [30].

In einer aktuellen retrospektiven Studie von Shim et al. hat Triptorelin im Vergleich zu den Agonisten Goserelin und Leuprorelin den Testosteronwert bei PCa-Patienten am effektivsten supprimiert [31]. Dieses könnte ein möglicher Erklärungsansatz für die positiveren Überlebensdaten für Triptorelin sein.

Die Mortalitätsrate über die Kohorte hinweg von fast einem Viertel der Patienten steht in Kontrast mit der Anzahl nicht metastasierter Patienten bei Studieneinschluss (70\%). Hier ist jedoch erneut die potenzielle Kodierungsungenauigkeit bei der Beurteilung der Abrechnungsdaten zu beachten sowie die Tat- sache, dass das allgemeine Versterben (Sterbeursache unklar) in den GKV-Daten dokumentiert und betrachtet wurde.

Wie die vorliegende Untersuchung zeigt, werden in Deutschland 85->90\% aller GnHRa von Urologen verordnet. In einer europaweiten Marktanalyse (Prostate Cancer Market Sizing, internal report 2016, Kantar Health und Ipsen) ergibt sich ein etwas anderes Bild: Es werden beim hormonsensitiven PCa ca. $70 \%$ der GnRHa von Urologen, $20 \%$ von Onkologen und fast $10 \%$ von Radiotherapeuten verordnet. Diese Verteilung verschiebt sich mit Fortschreiten der Erkrankung, sodass beim kastrationsresistenten PCa>50\% der GnRHa-Verschreibungen von Onkologen, nur noch ca. $40 \%$ von Urologen und weiterhin etwa $10 \%$ von Radio-Onkologen erfolgen. Das in der hier vorgestellten Analyse gezeigte Verschreibungsverhalten deutet in Deutschland hingegen auf eine vorwiegende Betreuung durch Urologen hin.

In Bezug auf notwendige Krankenhausaufnahmen fällt die deutlich unter dem Durchschnitt liegende Hospitalisierungsrate von Patienten mit Triptorelin auf. Im Gegensatz dazu verzeichnete die Degarelix-Kohorte statistisch signifikant mehr Hospitalisierungen, was auf den unter Degarelix vergleichsweise höheren Anteil metastasierter Patienten und einer hohen Inzidenz von obstruktiver Uropathie zurückzuführen sein könnte. Auch die stationären Ausgaben waren für Patienten mit Triptorelin verglichen mit den anderen Kohorten am geringsten, vermutlich aufgrund von Einsparungen infolge einer niedrigeren Hospitalisierungsrate. Diese Tendenz wird durch das Ergebnis der Benchmarkpopulation untermauert.

Die Limitationen der Studie sind bereits zu Beginn der Diskussion erwähnt. Neben Krankenkassendaten können auch Landeskrebsregister oder Selbsthilfegruppen als wichtige Informationsquellen für eine bedeutsame und aussagekräftige Abbildung der Versorgungsrealität dienen.

\section{Schlussfolgerung}

Anhand der Ergebnisse dieser vergleichenden retrospektiven Analyse über die GnRHa-Versorgungsrealität beim fortgeschrittenen PCa in Deutschland konnten Unterschiede hinsichtlich Verordnungsmuster, Hospitalisierungsraten und Krankenhauskosten aufgezeigt werden. Unter Triptorelin sind die geringste Hospitalisierungsrate, die kürzeste Krankenhausverweildauer sowie die niedrigsten -Gesamtkosten zu verzeichnen.

\section{Danksagung}

Die Autoren danken Dr. Juliane Schreier (co.medical, Berlin, Deutschland) für das Medical Writing, das von Ipsen Pharma GmbH (München, Deutschland) gemäß der Good Publication Practice Leitlinie gesponsert wurde. 
Interessenkonflikt

Finanzierung: Diese Arbeit wurde von der Ipsen Pharma GmbH, München, Deutschland, unterstützt. MH: Reisekosten/Honorar von Ipsen. PH: Sprecher/Berater für Janssen, Ipsen, Takeda, and Sanofi. MK: keine. NK: keine. CC: Angestellte von Ipsen Pharma GmbH, München. AM: Sprecher/Berater für Astellas, BMS, Merck, Ferring, Roche, Janssen, Ipsen, Takeda, und Sanofi

Literatur

[1] Kaatsch P, Spix C, Katalinic A et al. Krebs in Deutschland für 2013/ 2014. Berlin: Robert Koch-Institut und die Gesellschaft der epidemiologischen Krebsregister in Deutschland e. V; 2017

[2] Wong MC, Goggins WB, Wang HH et al. Global Incidence and Mortality for Prostate Cancer: Analysis of Temporal Patterns and Trends in 36 Countries. European urology 2016; 70: 862-874

[3] Mottet N, van den Bergh R, Briers E. Guidelines on prostate cancer. 2018: Available at: https://uroweb.org/guideline/prostate-cancer/

[4] Hinz SB. Monitoring of treatment for metastatic castration-resistant prostate cancer. Aktuelle Urologie 2017; 48: 225-229

[5] Huggins C, Hodges CV. Studies on prostatic cancer. I. The effect of castration, of estrogen and androgen injection on serum phosphatases in metastatic carcinoma of the prostate. CA: a cancer journal for clinicians 1972; 22: 232-240

[6] Cornford P, Bellmunt ], Bolla M et al. EAU-ESTRO-SIOG Guidelines on Prostate Cancer. Part II: Treatment of Relapsing, Metastatic, and Castration-Resistant Prostate Cancer. European urology 2017; 71: 630642

[7] Deutsche Krebsgesellschaft, Deusche Krebshilfe, AWMF. Leitlinienprogrogramm Onkologie: Interdisziplinäre Leitlinie der Qualität S3 zur Früherkennung, Diagnose und Therapie der verschiedenen Stadien des Prostatakarzinoms, Langversion 5.0, AWMF Registernummer: 043/022OL. 2018: https://www.leitlinienprogramm-onkologie. de/leitlinien/prostatakarzinom/ (last accessed on: 26/11/2018)

[8] Merseburger AS, Hupe MC. An Update on Triptorelin: Current Thinking on Androgen Deprivation Therapy for Prostate Cancer. Advances in therapy 2016; 33: 1072-1093

[9] Shore ND. Experience with degarelix in the treatment of prostate cancer. Therapeutic advances in urology 2013; 5: 11-24

[10] Furr B], Tucker $\mathrm{H}$. The preclinical development of bicalutamide: pharmacodynamics and mechanism of action. Urology 1996; 47: 13-25; discussion 29-32

[11] Sufrin G, Coffey DS. Flutamide. Mechanism of action of a new nonsteroidal antiandrogen. Investigative urology 1976; 13: 429-434

[12] Ryan C], Smith MR, de Bono JS et al. Abiraterone in metastatic prostate cancer without previous chemotherapy. The New England journal of medicine 2013; 368: 138-148

[13] Scher HI, Fizazi K, Saad F et al. Increased survival with enzalutamide in prostate cancer after chemotherapy. The New England journal of medicine 2012; 367: 1187-1197

[14] Rydzewska LHM, Burdett S, Vale CL et al. Adding abiraterone to androgen deprivation therapy in men with metastatic hormone-sensitive prostate cancer: A systematic review and meta-analysis. European journal of cancer (Oxford, England : 1990) 2017; 84: 88-101

[15] Smith MR, Saad F, Chowdhury S et al. Apalutamide Treatment and Metastasis-free Survival in Prostate Cancer. The New England journal of medicine 2018: doi:10.1056/NEJMoa1715546
[16] Hussain M, Fizazi K, Saad F et al. Enzalutamide in Men with Nonmetastatic, Castration-Resistant Prostate Cancer. The New England journal of medicine 2018; 378: 2465-2474

[17] Merseburger AS, Hammerer P, Rozet F et al. Androgen deprivation therapy in castrate-resistant prostate cancer: how important is $\mathrm{GnRH}$ agonist backbone therapy? World journal of urology 2015; 33: 10791085

[18] Hupe MC, Hammerer P, Ketz M et al. Retrospective analysis of patients with prostate cancer initiating $\mathrm{GnRH}$ agonists/antagonists therapy using a German claims database: epidemiological and patient outcomes. Frontiers in Oncology: Genitourinary Oncology 2018: doi:10.3389/fonc. 2018.00543

[19] Andersohn F, Walker J. Characteristics and external validity of the German Health Risk Institute (HRI) Database. Pharmacoepidemiology and drug safety 2016; 25: 106-109

[20] Schulze M, Stiegler H, Thielecke $C$ et al. Cross-sectional analysis of routine treatment for prostate cancer patients: CAPRIS - a healthcare research project of the IQUO. Der Urologe Ausg A 2014; 53: 865-870

[21] Bolla M, Van Tienhoven G, Warde P et al. External irradiation with or without long-term androgen suppression for prostate cancer with high metastatic risk: 10-year results of an EORTC randomised study. The Lancet Oncology 2010; 11: 1066-1073

[22] Efstathiou JA, Bae K, Shipley WU et al. Cardiovascular mortality after androgen deprivation therapy for locally advanced prostate cancer: RTOG 85-31. Journal of clinical oncology : official journal of the American Society of Clinical Oncology 2009; 27: 92-99

[23] Keating NL, O'Malley A], Freedland S] et al. Diabetes and cardiovascular disease during androgen deprivation therapy: observational study of veterans with prostate cancer. Journal of the National Cancer Institute 2010; 102: 39-46

[24] Tschope C, Kherad B, Spillmann F et al. Cardiovascular risk of androgen deprivation therapy for treatment of hormone-dependent prostate cancer : Differences between GnRH antagonists and GnRH agonists. Herz 2016; 41: 697-705

[25] Merseburger AS, Sedding D, Huter K. Cardiovascular risk patients under androgen deprivation therapy: Lower risk with $\mathrm{GnRH}$ antagonists compared to LHRH agonists? Der Urologe Ausg A 2016; 55: 218-225

[26] Merseburger A, Bro FalkenbergA, Kornilova OJ. New study suggests patients with advanced prostate cancer on androgen deprivation therapy need more dialogue with health care provider, especially around cardiovascular risk. World journal of urology 2018: doi:10.1007/s00345-018-2495-0

[27] Scailteux L-M, Vincendeau S, Balusson F et al. Androgen deprivation therapy and cardiovascular risk: No meaningful difference between $\mathrm{GnRH}$ antagonist and agonists-a nationwide population-based cohort study based on 2010-2013 French Health Insurance data. European Journal of Cancer 2017; 77: 99-108

[28] Varenhorst E, Klaff R, Berglund A et al. Predictors of early androgen deprivation treatment failure in prostate cancer with bone metastases. Cancer medicine 2016; 5: 407-414

[29] Heyns CF, Simonin MP, Grosgurin P et al. Comparative efficacy of triptorelin pamoate and leuprolide acetate in men with advanced prostate cancer. BJU international 2003; 92: 226-231

[30] Uttley L, Whyte S, Gomersall T et al. Degarelix for Treating Advanced Hormone-Dependent Prostate Cancer: An Evidence Review Group Perspective of a NICE Single Technology Appraisal. PharmacoEconomics 2017; 35: 717-726

[31] Shim M, Bang WJ, Oh CY et al. Effectiveness of three different luteinizing hormone-releasing hormone agonists in the chemical castration of patients with prostate cancer: Goserelin versus triptorelin versus leuprolide. Investigative and clinical urology 2019; 60: 244-250 